\title{
Sequential FDG-PET and induction chemotherapy in locally advanced adenocarcinoma of the Oesophago-gastric junction (AEG): The Heidelberg Imaging program in Cancer of the oesophago-gastric junction during Neoadjuvant treatment: HICON trial
}

Sylvie Lorenzen ${ }^{1 *}$, Carl von Gall², Annika Stange1, Georg M Haag ${ }^{1}$, Jürgen Weitz³ , Uwe Haberkorn², Florian Lordick ${ }^{4}$, Wilko Weichert ${ }^{5}$, Ulrich Abel ${ }^{6}$, Jürgen Debus ${ }^{7}$, Dirk Jäger ${ }^{1}$ and Marc W Münter ${ }^{7}$

\begin{abstract}
Background: 18-Fluorodeoxyglucose-PET ( $\left({ }^{18} \mathrm{~F}\right.$-FDG-PET) can be used for early response assessment in patients with locally advanced adenocarcinomas of the oesophagogastric junction (AEG) undergoing neoadjuvant chemotherapy. It has been recently shown in the MUNICON trials that response-guided treatment algorithms based on early changes of the FDG tumor uptake detected by PET are feasible and that they can be implemented into clinical practice.

Only $40 \%-50 \%$ of the patients respond metabolically to therapy. As metabolic non-response is known to be associated with a dismal prognosis, metabolic non-responders are increasingly treated with alternative neoadjuvant chemotherapies or chemoradiation in order to improve their clinical outcome. We plan to investigate whether PET can be used as response assessment during radiochemotherapy given as salvage treatment in early metabolic nonresponders to standard chemotherapy.
\end{abstract}

Methods/Design: The HICON trial is a prospective, non-randomized, explorative imaging study evaluating the value of PET as a predictor of histopathological response in metabolic non-responders. Patients with resectable AEG type I and II according to Siewerts classification, staged cT3/4 and/or cN+ and cMO by endoscopic ultrasound, spiral CT or MRI and FDG-PET are eligible. Tumors must be potentially RO resectable and must have a sufficient FDG-baseline uptake. Only metabolic non-responders, showing a $<35 \%$ decrease of SUV two weeks after the start of neoadjuvant chemotherapy are eligible for the study and are taken to intensified taxane-based RCT (chemoradiotherapy (45 Gy) before surgery. ${ }^{18}$ FDG-PET scans will be performed before ( = Baseline) and after 14 days of standard neoadjuvant therapy as well as after the first cycle of salvage docetaxel/cisplatin chemotherapy (PET 1) and at the end of radiochemotherapy (PET2). Tracer uptake will be assessed semiquantitatively using standardized uptake values (SUV). The percentage difference $\triangle S U V=100\left(S_{\text {Baseline }}\right.$ - SUV PET1)/SUV Baseline will be calculated and assessed as an early predictor of histopathological response. In a secondary analysis, the association between the difference SUV $V_{\text {PET1 }}$ - SUV PET2 and histopathological response will be evaluated.

\footnotetext{
* Correspondence: sylvielorenzen@gmx.de

'National Center for Tumor Diseases (NCT), University of Heidelberg,

Germany

Full list of author information is available at the end of the article
} 
Discussion: The aim of this study is to investigate the potential of sequential ${ }^{18} \mathrm{FDG}$-PET in predicting histopathological response in AEG tumors to salvage neoadjuvant radiochemotherapy in patients who do not show metabolic response to standard neoadjuvant chemotherapy.

Trial Registration: Clinical trial identifier NCT01271322

\section{Background}

Oesophageal cancer is among the 10 most common malignancies worldwide and is associated with a high mortality $[1,2]$. Often, the tumors are locally advanced at the time of initial diagnosis because symptoms do not appear until late (T3-T4, N+, or M1). In cases of locally advanced tumors $(\mathrm{T} 3 / \mathrm{T} 4, \mathrm{~N}+)$, surgery remains the mainstay of therapy, but evidence is growing that preoperative chemotherapy or chemoradiotherapy improves survival in responding patients with locally advanced adenocarcinoma of the oesophagus and the oesophagogastric junction [3,4]. However, for patients who do not respond, the prognosis after neoadjuvant therapy might be worse than that of a primarily surgical approach [5]. These metabolic non-responders have a low histopathological response rate of only $5 \%$ and a poor prognosis compared with responders [6]. Since about half of the patients treated with neoadjuvant chemotherapy will not respond [7], an early predictor of response would avoid futile therapy and allow patients to pursue other, potentially more efficacious treatments. Therefore, an individual early assessment of response to neoadjuvant therapy using imaging techniques could be of great value for tailoring neoadjuvant treatment as well as the surgical approach to the individual patient [8-10]. Over the past few years, many attempts have been made to improve prognostication of the individual tumor biology in oesophageal carcinoma and to identify prognostic and predictive biomarkers.

Metabolic changes measured by PET have been shown to be more sensitive in detecting response early in the course of chemotherapy as compared with both conventional imaging techniques (EUS and CT) and endoscopy [11]. Various studies have demonstrated that 18-fluorodeoxyglucosepositron emission tomography (FDG-PET), measuring early changes in tumor glucose uptake after only two weeks of induction therapy, is a promising tool in the prediction of clinical and histopathologic response as well as prognosis to neoadjuvant treatment in adenocarcinomas of the oesophagogastric junction (AEG) type I and II $[6,12]$. Available evidence suggests that metabolic response might be a useful predictive marker for the early identification of nonresponding patients. The MUNICON-I trial prospectively showed that early metabolic assessment with therapy stratification after only 2 weeks helps to select patients who are not benefiting from neoadjuvant chemotherapy and can therefore avoid ineffective and toxic therapy in non- responding patients with AEG I and II [8,9]. These patients with poor prognosis and potentially biologically even more aggressive tumors might be suitable candidates for intensification of the neoadjuvant treatment, by changing the chemotherapeutic regimen in attempting to overcome chemoresistance, and by adding radiochemotherapy.

Evidence in the literature gives the rationale to use a taxane-based regimen in patients who are not responding to a first line EOX/EOF (Epirubicin, Oxaliplatin, Capecitabin/5-Fluorouracil) induction therapy [13]. A phase II trial showed that Docetaxel given with Cisplatin is a highly active chemotherapy schedule and permitted surgery in initially inoperable patients [14]. Furthermore, neoadjuvant chemoradiotherapy for adenocarcinoma of the oesophagus seems to improve overall survival [4] and may induce significantly higher response rates compared to chemotherapy alone [15].

The primary objective of the Heidelberg Imaging program in Cancer of the Oesophago-gastric junction during Neoadjuvant treatment ( $\mathrm{HICON}$ ) trial is to investigate whether semiquantitative measurements of ${ }^{18}$ F-FDG accumulation at the primary tumor site using (sequential) PET could be applied to assess the effects of salvage neoadjuvant radiochemotherapy in initially non-responding tumors, with metabolic response defined by a $\geq 35 \%$ decrease in ${ }^{18} \mathrm{~F}$-FDG uptake two weeks after induction chemotherapy $[6,12]$, using histopathology as a gold standard.

\section{Methods/Design}

\section{Study Design}

HICON is a prospective, non-randomized, exploratory imaging/biomarker study. The study is designed and coordinated by the National Center for Tumor Disease (NCT), Heidelberg. The protocol of the study was approved by the local Ethics Committee, and was also subject to authorization by the German radiation protection authority (Bundesamt für Strahlenschutz $=$ BfS) as mandatory by federal law.

All participants provide written informed consent.

The study was assigned the number NCT01271322 in the European Clinical Trials Database (EudraCT).

\section{Study objectives and endpoints}

The primary objective of the study is to evaluate the change in metabolic response as measured by the relative difference $\Delta \mathrm{SUV}=100\left(\mathrm{SUV}_{\text {Baseline }}-\mathrm{SUV}_{\mathrm{PET1}}\right) / \mathrm{SUV}_{\text {Baseline }}$ 
in ${ }^{18} \mathrm{~F}$-FDG uptake after 1 cycle of salvage taxan-based chemotherapy (PET1), relative to the ${ }^{18}$ F-FDG uptake at the baseline examination, as a predictor of histopathological response in initial metabolic non-responders (assessed by PET 14 days after the start of neoadjuvant therapy). Secondary objectives are the investigation of the distribution of $\triangle \mathrm{SUV}$ in histological responders and non-responders, the accuracy of the binary prediction rule $\triangle S U V$ $\geq 65 \%$ vs. $<65 \%$ (in particular the question whether this rule is superior to a random prediction), the association of SUV measured by PET1 with the value of PET response after intensified neoadjuvant radiochemotherapy (PET2), the association between SUV $\mathrm{PET}_{1}-\mathrm{SUV}_{\mathrm{PET} 2}$ and histopathological response, and the association between $\triangle \mathrm{SUV}$ and overall survival as well as disease-free survival (counted from the date of recruitment).

\section{Patient selection}

Eligibility criteria include the presence of biopsy-proven adenocarcinoma of the distal oesophagus (AEG type I) or cardia (AEG type II) [16], staged as CT3 or cT4, with or without metastases in local lymph nodes and no evidence of hematogenous metastases. Staging procedures include endoscopy, endoscopic ultrasound and computed tomography $(\mathrm{CT})$ of the chest and abdomen. Eligible patients have to be fit for platin-containing chemotherapy and tumors must be potentially $\mathrm{R} 0$ resectable during consecutive operation.

Tumors must have demonstrated a minimal amount of FDG-uptake in the baseline PET-CT, defined as ${ }^{18}$ FDG-uptake in tumor at first examination $>1,35 \times$ hepatic-SUV $+2 \times$ standard-deviation of hepatic-SUV, and must be a metabolic non-responder under EOX, defined as a decrease of the SUVmax of $<35 \%$ in a second PET on day 14 of chemotherapy.

Patients with an Eastern Cooperative Oncology Group score worse than 1, previous or secondary malignancy, life expectancy of less than 3 months, uncontrolled bleeding from the tumor, tumor infiltration of the airways, pregnancy, uncontrolled diabetes, or age less than 18 years are excluded. Patients are also ineligible if they have undergone previous chemotherapy, radiotherapy, or endoscopic laser therapy.

\section{Treatment schedule/follow-up}

After obtaining written informed consent, a baseline FDG-PET will be performed during initial staging within one week before initiation of preoperative chemotherapy. Patients will be eligible for inclusion only if PET scans show a sufficient contrast between tumor and surrounding tissues: this is based on standard uptake value (SUV) measurements. All eligible patients will be given the following induction chemotherapy:

EOX: Epirubicin $50 \mathrm{mg} / \mathrm{m}^{2}$ i.v. Bolus
Oxaliplatin $130 \mathrm{mg} / \mathrm{m}^{2}$ i.v.

Capecitabin $2 \times 625 \mathrm{mg} / \mathrm{m}^{2}$ p.o.

d1-21

Or (only if dysphagia):

EOF: Epirubicin $50 \mathrm{mg} / \mathrm{m}^{2}$ i.v. Bolus

Oxaliplatin $130 \mathrm{mg} / \mathrm{m}^{2}$ i.v.

d1

5-FU $200 \mathrm{mg} / \mathrm{m}^{2} / \mathrm{d}$ continuously i.v. d1-21

\section{FDG-PET will be repeated on day 14 of the first chemotherapy cycle}

Patients whose tumor SUV will be decreased by $\geq 35 \%$, will be defined as metabolic responders. This cut-off is predefined and is based on previous research $[6,8,12]$. Metabolic responders will continue to receive chemotherapy for a maximum of 12 weeks before surgery.

Metabolic non-responders, with a SUV decrease of less than 35\%, discontinue induction chemotherapy and proceed to an intensified salvage radiochemotherapy treatment, which forms the basis of the HICON study (see Figure 1). Within this imaging/biomarker study, patients will receive two more FDG-PET assessments to evaluate metabolic response: the first right before the onset of radiochemotherapy (PET1) and the second after completion of radiochemotherapy and prior to resection (PET2).

Within the HICON study, patients will be treated with one cycle of chemotherapy with docetaxel $75 \mathrm{mg} / \mathrm{m}^{2}$, and cisplatin $75 \mathrm{mg} / \mathrm{m}^{2}$ to allow radiation planning.

This treatment will be followed by radiochemotherapy, starting on day 22 of intensified chemotherapy cycle:

\section{Radiotherapy}

$1.8 \mathrm{~Gy} /$ day, total dose $45 \mathrm{~Gy}$

Docetaxel

$25 \mathrm{mg} / \mathrm{m}^{2}$ on days $1,8,15,22,29$ of radiochemotherapy (RCT) and Cisplatin: $25 \mathrm{mg} / \mathrm{m}^{2}$ on days $1,8,15,22,29$ of RCT. Docetaxel should be given before cisplatin.

Chemotherapy is given on a weekly basis. Combined radiochemotherapy, with a total dose of $45 \mathrm{~Gy}$, is followed by resection, 28 - 42 days after the end of RCT. The protocol treatment ends 30 days post resection, or if a longer hospital stay is needed, until the last day of hospitalization.

After surgical resection, patients will be followed at 3month intervals by $\mathrm{CT}$ of the chest and abdomen and upper intestinal endoscopy. In patients with curative (R0) resection, time to recurrence will be calculated as the time from initiation of neoadjuvant therapy to detection of local recurrence or distant metastases. In patients with no resection or residual tumor after resection ( $\mathrm{R} 1$ and $\mathrm{R} 2$ resection, respectively), time to tumor progression will be determined.

The duration of the study is anticipated to be 15 months (10 months accrual time plus 5 months of individual follow-up, ending with the assessment of histopathological response). After the end of study participation, patients 


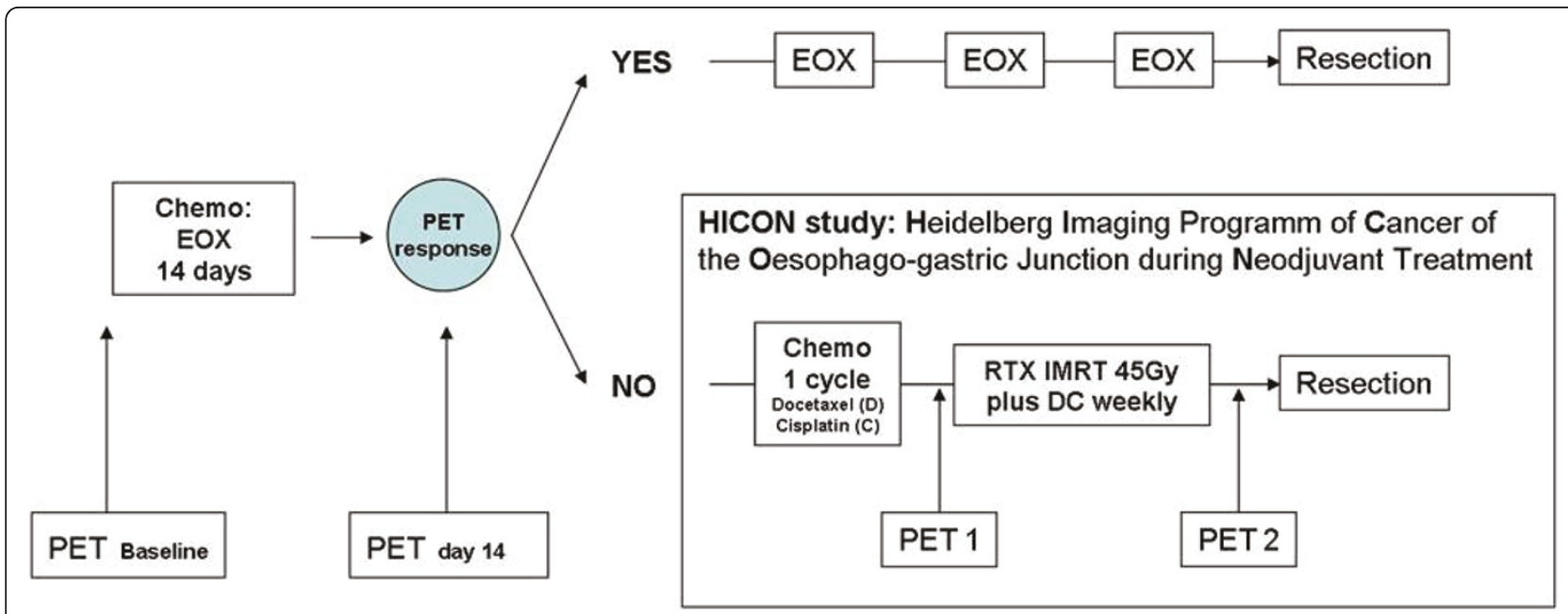

Figure 1 Trial flow chart.

will be followed according to the standard follow-up scheme implemented at the NCT Heidelberg. Data on survival and relapse gathered during this routine follow-up will be used for the assessment of survival and disease-free survival for study patients.

\section{Supportive therapy}

Proton-pump inhibitors (PPIs) will be given once daily in standard dosage during the entire preoperative period.

Premedication with dexamethasone will be performed according to local standards.

Antiemetic prophylaxis and therapy is recommended according to local standards.

Granulocyte-Colony Stimulating Factor (G-CSF): In the case of neutrophils nadir $<0.5 \times 10^{9} / 1$ during the previous cycle, G-CSF may be administered during the following cycles according to local standards.

\section{Hydration}

During CT and RCT, standard i.v. hydration before and after cisplatin treatment is mandatory, according to local rules. Hydration during weekly cisplatin will be done according to local standards.

\section{PET Imaging}

An ${ }^{18}$ F-FDG PET/CT scan will be performed for each patient before the initiation of preoperative chemotherapy (baseline PET) and 14 days after the initiation of chemotherapy (PET day14). In metabolically nonresponding patients ( $<35 \%$ SUV decrease), a third PET scan after initiation of taxan-based chemotherapy and right before radiochemotherapy (PET1) and a fourth PET scan after the completion of radiochemotherapy and immediately before surgery (PET2) will be performed.
PET scans will be done using a Biograph 6 (Siemens AG, Erlangen, Germany), axial field of view of $15.4 \mathrm{~cm}$ in 3D mode. Patients will fast at least 6 hours before PET imaging to ensure euglycamic glucose metabolism. Blood glucose levels are measured before each PET scan. Prior to each application, patients are advised to rest and continue to reduce activities for 50 minutes after administration of 250 to $350 \mathrm{MBq}$ 18F-FDG followed by $500 \mathrm{ml}$ saline solution to increase the distribution volume.

60 minutes post injection a whole body scan with 3 $\mathrm{min} / \mathrm{BP}$ from head to upper femur is performed.

The reconstruction of the raw images is performed after dead time, scatter and random correction using an iterative method based on the ordered subsets expectation maximization algorithm (OSEM) of four iterations/ 8 subsets, $256 \times 256$ matrix, Gaussian smoothing and of $3.5 \mathrm{~mm}$ transversal slices at full with half maximum. The image data set is normalized for the injected dose and the patients body weight, resulting in parametric imaging using standardized uptake value (SUV) on the basis of the formula "SUV = tissue concentration $(\mathrm{Bq} /$ $\mathrm{g}) /($ injected dose $(\mathrm{Bq}) /$ body weight $(\mathrm{g}))$ ".

The spatial resolution of the reconstructed images is 6 to $8 \mathrm{~mm}$ at full width half maximum. For quantitative evaluation, an automated volume of interest (VOI) derived from generated regions of interst (ROI) using the auto3D function within the Syngo Software (Siemens AG, Erlangen, Germany) will be placed over the tumor in the slice with maximum FDG uptake in the baseline scan. In the second PET scan, the region of interest will be placed at the same position as in the baseline PET as a reference. For patients treated in the HICON study, the change of FDG-uptake before (PET1) and after (PET2) radiochemotherapy will be assessed in relation to the baseline PET. 
For further analysis the mean value of the two measurements is used.

\section{Preoperative Radiochemotherapy}

The treatment dosage of this radiochemotherapy scheme is based upon a SAKK study (SAKK 75/02), which purpose was to investigate the efficacy, toxicity, and feasibility of preoperative docetaxel-cisplatin together with radiotherapy. This trimodality treatment showed feasibility in combination with favourable antineoplastic activity and survival data compared with other trials [17].

After positioning the patient in an adequate custommade device a treatment planning $\mathrm{CT}$ will be performed. In all cases a contrast-enhanced CT with a thickness of $3 \mathrm{~mm}$ is required. The treatment planning CT scan should be acquired with the patient in the same position and using the same immobilization device as for treatment. All tissues receiving irradiation should be included in the CT scan. A slice by slice segmentation of the target volume and all organs at risk will be performed. Gross Tumor Volume (GTV) represents the region judged to contain gross primary tumor or involved node(s) based on CT scan and other imaging techniques. The Clinical Target Volume (CTV) is defined as the GTV plus areas considered at risk for containing microscopic disease delineated by the treating physician. CTV represents the GTV plus a margin of generally $0.2 \mathrm{~cm}$ and the nodal regions receiving elective irradiation. The CTV margins can be narrower when GTV is in the proximity of the spinal cord or critical normal tissues. All efforts should be made to adapt and delineate the CTV according to the location of the primary tumor and the relative incidence of lymph node involvement in the different anatomic regions of the lymph nodes. Planning Target Volume (PTV) represents an additional margin of at least $0.2 \mathrm{~cm}$ around the CTV to compensate for the variability of treatment set up and internal organ motion.

The applied total dose is 45 Gy in 25 fractions (1.8 Gy single dose). Therefore the radio-therapy will be applied in 5 weeks. For the normal tissue the tolerance doses (TD 5/5) defined by Emami et al. [18] will be applied. At least $95 \%$ of the CTV should receive $90 \%$ of the prescribed total dose and the maximum dose should be smaller than $110 \%$.

For treatment a linear accelerator with at least $6 \mathrm{MeV}$ photons will be used. Intensity modulated radiation therapy (IMRT) is recommended but according to the treatment protocol also 3-D planned treatment techniques are allowed. The linear accelerator should be equipped with image guidance (IGRT) and at least weekly positioning controls will be performed. In case of severe deviations in the positioning of the patients new treatment plans should be calculated.

\section{Surgical Therapy}

The recommended surgical procedure in patients with AEG type I is an abdominothoracic oesophagectomy with intrathoracic anastomosis. In patients with AEG type II tumors, a transhiatal extended gastrectomy and an extended D2-lymphadenectomy, including a left retroperitoneal lymphadenectomy can be performed [19].

\section{Histopathologic Analysis}

For assessment of histopathologic tumor regression, the resected primary tumors will be evaluated according to a recently published scoring system [20]. All patients with less than $10 \%$ residual tumor (regression score $1 \mathrm{a}$ and $1 \mathrm{~b}$ ) will be classified as responders. All other tumors (regression score 2 and 3 ) will be classified as nonresponders.

\section{Statistical considerations and sample size estimation}

The sample size/power calculations were based on a univariate logistic regression analysis of the predicitveness of $\triangle \mathrm{SUV}$, for histopathological response. The projected sample size of 25 evaluable patients is sufficient to detect, with a power of $>80 \%$, an increase of the AUC of the ROC curve of $\triangle S U V$ (with respect to histopathological response) from 0.5 to 0.8 , assuming that the values of $\triangle \mathrm{SUV}$ in the groups $\mathrm{R}$ and $\mathrm{N}$ are represented by independent normally distributed variables with equal variances, and assuming a histopathological response rate of $\mathrm{r}=50 \%$ (calculations based on 10000 computer stimulations, $\alpha=$ $10 \%)$. While, under the assumptions made above the study was slightly overpowered (estimated power $=84.7 \%$ ), the sample size of $\mathrm{n}=25$ to some extent safeguards against the loss of power occurring if $r \neq 50 \%$, with, e.g., an estimated power of $80.3 \%$ resulting for $r=65 \%$. Assuming a drop-out rate of about $10 \%$ the total number of patients to be recruited was set at $\mathrm{n}=28$.

Demographic and other baseline variables, as well as the treatment-related variables (like, e.g., the number of treatment cycles, dosages, dose modifications) will be analyzed using frequency tables in case of categorical variables and summary statistics for quantitative variables. The results of PET scans will be described by means of summary statistics, box plots, and scatter plots, both overall and for histological responders and non-responders separately. The analysis of the primary endpoint will be done using univariate logistic regression with $\triangle \mathrm{SUV}$ (or a monotone transform of $\Delta \mathrm{SUV}$ ) as the predictor variable and histological response as the outcome variable. In this analysis, the statistical significance level is set at $\alpha=10 \%$. The accuracy of the binary prediciton rule $\Delta \mathrm{SUV} \geq 65 \%$ vs. $<65 \%$ will be estimated together with Clopper-Pearson 95\% confidence bounds. Fisher's exact test will be used to test to compare the accuracy of this rule with a random prediction. The association between the difference $S U V_{\text {PET1 }}$ and SUV $V_{\text {PET2 }}$ 
will be examined by calculating Spearman's rank correlation coefficient and by analyzing the agreement according to the method proposed by Bland and Altman [21]. The agreement of the results of the aformentioned binary rule applied to both SUV PET1 and SUV ${ }_{\text {PET2 }}$ will be assessed by means of McNemar's test. The association between the difference D12 $=\mathrm{SUV}_{\mathrm{PET} 1}-\mathrm{SUV}_{\mathrm{PET} 2}$ and histopathological response will be analyzed by means of the Wilcoxon rank sum test comparing histopathological responders and non-responders. A ROC curve for D12 with respect to response will be produced. The association between $\triangle \mathrm{SUV}$ and overall survival as well as disease-free survival will be assessed using the Cox Proportional Hazard model. Safety will be analyzed based on adverse evnts and shift tables for laboratory parameters.

Except for the logistic regression analysis of the primary endpoint, all analyses are strictly exploratory. For each particular objective and endpoint, the analysis population consists of all patients for whom the measurements required for the analysis are available. Patients having no PET1-measurement or who drop out before the histopathological response has been determined will be replaced. No interim analyses will be carried out.

\section{Discussion}

The most exciting use of FDG-PET in the management of localized gastroesophageal cancer is the early assessment of metabolic response during neoadjuvant chemotherapy. In this indication, early metabolic response assessment has been shown to contribute to the individualization of treatment algorithms; cut-off values have been prospectively validated and have also been used in interventional clinical studies $[6,8,10,22,23]$.

As metabolically non-responding patients have poor survival chances even after R0 resection, the early identification of these non-responding tumors by FDG-PET may allow an adaption and optimization of preoperative treatment. We hypothesize that non-responding patients after only 2 weeks of chemotherapy might benefit from a change of the chemotherapy regimen and the additional use of radiotherapy.

In previous studies, metabolic non-response was associated with histopathological non-response [8], however it remains to be determined if subsequent radiochemotherapy can transform some metabolic non-responders after induction chemotherapy into histopathological responders.

While the utility of FDG-PET for early response assessment has been shown after neoadjuvant chemotherapy alone, the role of FDG-PET in monitoring the antitumoral activity of radiochemotherapy has not been established.

This study was designed to assess the value of metabolic response evaluation before and after initiation of salvage radiochemotherapy (RCT) in patients with metabolic non-response after 2 weeks of induction chemotherapy. In order to overcome chemoresistance, the neo-adjuvant treatment regimen will be intensified by changing the chemotherapeutic agent and adding radiation treatment.

However, this study is only designed as an imaging/ biomarker study, and further clinical trials are necessary to eventually answer the question of whether further neoadjuvant chemotherapy or chemoradiation for ${ }^{18} \mathrm{~F}$ FDG PET non-responders improves their clinical outcome.

In this context it still needs to be evaluated whether a metabolic response fully captures the net effect of treatment on the clinical outcome.

Only randomized trials will ultimately be able to demonstrate whether treatment regimens with a higher metabolic response rate also lead to improved patient survival.

The European Organization of Research and Treatment of Cancer (EORTC) is currently planning a randomized international multicenter trial, comparing the activity of intensified taxane based RCT to immediate resection in PET non-responders in terms of histopathological response and survival.

The HICON study was designed to prospectively evaluate if quantitative assessment of tumor metabolism by FDG-PET is an efficient and clinically useful technique to monitor tumor response to salvage (radio)-chemotherapy early in the course of treatment.

In conclusion, this study aims to quantify metabolic tumor response by determining whether changes in tumor FDG-uptake, before and after intensified radiochemotherapy can predict histopathological response after completion of neoadjuvant therapy in initially metabolically non-responding patients.

If it would be possible to early select patients who will not benefit from intensified salvage radiochemotherapy, these patients could be spared intensified and potentially toxic salvage treatments which may impair quality of life; alternatively, patients could proceed directly to surgery.

\section{Author details \\ ${ }^{1}$ National Center for Tumor Diseases (NCT), University of Heidelberg, Germany. ${ }^{2}$ Department of Nuclear Medicine, University of Heidelberg, Germany. ${ }^{3}$ Department of Surgery, University Hospital of Heidelberg, Germany. ${ }^{4}$ Medizinische Klinik III, Klinikum Braunschweig, Germany. ${ }^{5}$ Institute of Pathology, University of Heidelberg, Germany. ${ }^{6}$ Department of Medical Biometry, University of Heidelberg, Germany. ${ }^{7}$ Department of Radiation Oncology, University of Heidelberg, Germany.}

\section{Authors' contributions}

$\mathrm{SL}, \mathrm{FL}, \mathrm{CVG}, \mathrm{AS}, \mathrm{MWM}, \mathrm{WW}, \mathrm{JD}, \mathrm{DJ}, \mathrm{UH}, \mathrm{JW}$ participated in the design of the study, UA was responsible for the statistical planning of the trial, AS and FL wrote the study protocol. SL and GMH are reponsible for conducting and 
co-ordination of the trial as well as patient recruitment. All authors read and approved the final manuscript.

\section{Competing interests}

The authors declare that they have no competing interests.

Received: 22 March 2011 Accepted: 24 June 2011

Published: 24 June 2011

\section{References}

1. Parkin DM: International variation. Oncogene 2004, 23(38):6329-6340.

2. Jemal A, Murray T, Ward E, Samuels A, Tiwari RC, Ghafoor A, Feuer EJ, Thun MJ: Cancer statistics, 2005. CA Cancer J Clin 2005, 55(1):10-30.

3. Cunningham D, Allum WH, Stenning SP, Thompson JN, Van de Velde CJ, Nicolson M, Scarffe JH, Lofts FJ, Falk SJ, Iveson TJ, et al: Perioperative chemotherapy versus surgery alone for resectable gastroesophageal cancer. N Engl J Med 2006, 355(1):11-20.

4. Gebski V, Burmeister B, Smithers BM, Foo K, Zalcberg J, Simes J: Survival benefits from neoadjuvant chemoradiotherapy or chemotherapy in oesophageal carcinoma: a meta-analysis. Lancet Oncol 2007, 8(3):226-234

5. Kelsen DP, Ginsberg R, Pajak TF, Sheahan DG, Gunderson L, Mortimer J, Estes N, Haller DG, Ajani J, Kocha W, et al: Chemotherapy followed by surgery compared with surgery alone for localized esophageal cancer. $N$ Engl J Med 1998, 339(27):1979-1984.

6. Ott K, Weber WA, Lordick F, Becker K, Busch R, Herrmann K, Wieder H, Fink U, Schwaiger M, Siewert JR: Metabolic imaging predicts response, survival, and recurrence in adenocarcinomas of the esophagogastric junction. J Clin Oncol 2006, 24(29):4692-4698.

7. Samalin E, Ychou M: Neoadjuvant treatment in upper gastrointestinal adenocarcinomas: new paradigms from old concepts? Curr Opin Oncol 2007, 19(4):384-389.

8. Lordick F, Ott K, Krause BJ, Weber WA, Becker K, Stein HJ, Lorenzen S, Schuster $T$, Wieder $H$, Herrmann $K$, et al: PET to assess early metabolic response and to guide treatment of adenocarcinoma of the oesophagogastric junction: the MUNICON phase II trial. Lancet Oncol 2007, 8(9):797-805.

9. Siewert JR, Lordick F, Ott K, Stein HJ, Weber WA, Becker K, Peschel C, Fink U, Schwaiger M: Induction chemotherapy in Barrett cancer: influence on surgical risk and outcome. Ann Surg 2007, 246(4):624-628, discussion 628-631.

10. Lordick F, Meyer Zum Bueschenfelde C, Herrmann K: PET-guided treatment in locally advanced adenocarcinoma of the esophagogastric junction (AEG): The MUNICON-II study. American Society of Clinical Oncology Gl Cancer Symposium 2011 2011, San Francisco CA (J Clin Oncol 29: 2011 (suppl 4; abstr 3)).

11. Wieder HA, Beer AJ, Lordick F, Ott K, Fischer M, Rummeny EJ, Ziegler S, Siewer JR, Schwaiger M, Weber WA: Comparison of changes in tumor metabolic activity and tumor size during chemotherapy of adenocarcinomas of the esophagogastric junction. J Nucl Med 2005, 46(12):2029-2034.

12. Weber WA, Ott K, Becker K, Dittler HJ, Helmberger H, Avril NE, Meisetschlager G, Busch R, Siewert JR, Schwaiger $M$, et al: Prediction of response to preoperative chemotherapy in adenocarcinomas of the esophagogastric junction by metabolic imaging. J Clin Oncol 2001, 19(12):3058-3065.

13. Roth AD, Fazio N, Stupp R, Falk S, Bernhard J, Saletti P, Koberle D, Borner MM, Rufibach K, Maibach R, et al: Docetaxel, cisplatin, and fluorouracil; docetaxel and cisplatin; and epirubicin, cisplatin, and fluorouracil as systemic treatment for advanced gastric carcinoma: a randomized phase II trial of the Swiss Group for Clinical Cancer Research. J Clin Oncol 2007, 25(22):3217-3223.

14. Lorenzen $S$, Hentrich $M$, Haberl $C$, Heinemann $V$, Schuster $T$, Seroneit $T$, Roethling N, Peschel C, Lordick F: Split-dose docetaxel, cisplatin and leucovorin/fluorouracil as first-line therapy in advanced gastric cancer and adenocarcinoma of the gastroesophageal junction: results of a phase II trial. Ann Oncol 2007, 18(10):1673-1679.

15. Stahl M, Walz MK, Stuschke M, Lehmann N, Meyer HJ, Riera-Knorrenschild J, Langer P, Engenhart-Cabillic R, Bitzer M, Konigsrainer A, et al: Phase III comparison of preoperative chemotherapy compared with chemoradiotherapy in patients with locally advanced adenocarcinoma of the esophagogastric junction. J Clin Oncol 2009, 27(6):851-856.
16. Siewert JR, Stein HJ: Classification of adenocarcinoma of the oesophagogastric junction. Br J Surg 1998, 85(11):1457-1459.

17. Ruhstaller T, Widmer L, Schuller JC, Roth A, Hess V, Mingrone W, von Moos R, Borner M, Pestalozzi BC, Balmermajno S, et al: Multicenter phase II trial of preoperative induction chemotherapy followed by chemoradiation with docetaxel and cisplatin for locally advanced esophageal carcinoma (SAKK 75/02). Ann Oncol 2009, 20(9):1522-1528.

18. Emami B, Lyman J, Brown A, Coia L, Goitein M, Munzenrider JE, Shank B, Solin $L$, Wesson M: Tolerance of normal tissue to therapeutic irradiation. Int J Radiat Oncol Biol Phys 1991, 21(1):109-122.

19. Ott K, Bader FG, Lordick F, Feith M, Bartels H, Siewert JR: Surgical factors influence the outcome after Ivor-Lewis esophagectomy with intrathoracic anastomosis for adenocarcinoma of the esophagogastric junction: a consecutive series of 240 patients at an experienced center. Ann Surg Oncol 2009, 16(4):1017-1025.

20. Becker K, Mueller JD, Schulmacher C, Ott K, Fink U, Busch R, Bottcher K, Siewert JR, Hofler $\mathrm{H}$ : Histomorphology and grading of regression in gastric carcinoma treated with neoadjuvant chemotherapy. Cancer 2003, 98(7):1521-1530

21. Bland JM, Altman DG: Statistical methods for assessing agreement between two methods of clinical measurement. Lancet 1986, 1(8476):307-310.

22. Rizk NP, Tang L, Adusumilli PS, Bains MS, Akhurst TJ, Ilson D, Goodman K, Rusch W: Predictive value of initial PET-SUVmax in patients with locally advanced esophageal and gastroesophageal junction adenocarcinoma. J Thorac Oncol 2009, 4(7):875-879.

23. Javeri $H$, Xiao L, Rohren E, Komaki R, Hofstetter W, Lee JH, Maru D, Bhutani MS, Swisher SG, Wang X, et al: Influence of the baseline 18Ffluoro-2-deoxy-D-glucose positron emission tomography results on survival and pathologic response in patients with gastroesophageal cancer undergoing chemoradiation. Cancer 2009, 115(3):624-630.

\section{Pre-publication history}

The pre-publication history for this paper can be accessed here: http://www.biomedcentral.com/1471-2407/11/266/prepub

doi:10.1186/1471-2407-11-266

Cite this article as: Lorenzen et al:: Sequential FDG-PET and induction chemotherapy in locally advanced adenocarcinoma of the Oesophagogastric junction (AEG): The Heidelberg Imaging program in Cancer of the oesophago-gastric junction during Neoadjuvant treatment: HICON trial. BMC Cancer 2011 11:266.

\section{Submit your next manuscript to BioMed Central and take full advantage of:}

- Convenient online submission

- Thorough peer review

- No space constraints or color figure charges

- Immediate publication on acceptance

- Inclusion in PubMed, CAS, Scopus and Google Scholar

- Research which is freely available for redistribution

Submit your manuscript at www.biomedcentral.com/submit
C) Biomed Central 